\title{
COUPLING BETWEEN CORE AND RING MODES IN A MICROSTRUCTURED FIBER
}

\author{
Yu-Hsiang Cheng, Chin-Ping Yu, Chi-Wen Huang, Yean-Woei Kiang, Hung-chun Chang, \\ Hua-Kuang Liu, and C. C. Yang \\ Graduate Institute of Electro-Optical Engineering, Graduate Institute of Communication \\ Engineering, and Department of Electrical Engineering, National Taiwan University, \\ 1, Roosevelt Road, Section 4, Taipei, Taiwan, R.O.C. \\ (phone) 886-2-23657624 (fax) 886-2-23652637 (e-mail) ccy@cc.ee.ntu.edu.tw
}

Abstract-The propagation characteristics of the core and ring modes and the coupling behaviors between them under micro bending in a specially designed micro-structured fiber were numerically studied with the finite-difference method.

In this paper, we propose a novel design of micro-structured fiber with a solid core and a solid ring region. Surrounded by air holes, a core mode and one or more ring modes, all well guided, were identified. The relative sizes of the effective refractive index of the core and ring modes depended on the sizes of the core and ring region. Then, the coupling behaviors between the core and ring modes were evaluated under micro bending. The air-hole distribution of the micro-structured fiber is schematically shown in Fig. 1. Here, one can see that the air hole distribution forms a solid core and a solid ring region. The following parameters are designed: the air hole spacing $a=2.3 \mu \mathrm{m}$, the diameter of the outer-shell hole $d=0.6 \mathrm{a}$, the thickness of the solid ring region $b=0.9 \mathrm{a}$, and the air-hole diameter between the core and ring regions $d^{\prime}=0.6 \mathrm{a}$. The considered wavelength is fixed at $1550 \mathrm{~nm}$. Fig. 2 shows the close-up of the core mode intensity distribution. This mode has the effective refractive index as 1.41354 . Fig. 3 shows the intensity distribution of the first ring mode (the one with the largest effective refractive index). This mode has the effective refractive index as 1.41857 . There are totally three ring modes in this micro-structured fiber. Note that the effective refractive index of the ring mode shown in Fig. 3 is larger than that of the core mode. We studied the variations of effective refractive index of various modes with the ring thickness $b / a$ when $d^{\prime} / a=0.6$. We found that the effective index of the core mode did not significantly change over the concerned $b$ range. However, the effective refractive indices of ring modes increase with the solid ring thickness. For b/a larger than 0.7 , the effective indices of the ring modes are larger than that of the core mode. For smaller b/a, the trend is reversed. The ring mode number increases with the ring thickness. Up to three ring modes were found when $b / a=0.9$. Then, the variation of effective index with d'/a was investigated. The results were obtained with the assumption that $b / a=0.9$ when $d^{\prime} / a=0.6$. Also, the centers of the air holes between the core and the solid ring region were not changed when d'/a was varied. Hence, as d' increased, the sizes of the solid core and ring regions were reduced. Therefore, the effective indices of all modes decrease with d'/a. Then, we show the coupling behaviors between the core mode and the cladding modes under micro bending. The micro bending period was set intending to phase-match the core mode the ring mode 1 when $b / a=0.9$ and $d^{\prime} / a=0.6$. The coupling coefficient varies with fiber length in a period of only $155 \mu \mathrm{m}$ although the micro bending period is $310 \mu \mathrm{m}$. This result is due to the symmetric field distributions of both core and ring modes in this case. The amplitude of coupling coefficient variation is as large as $6400 / \mathrm{m}$. The variations of coupling coefficient amplitude between the core mode and various ring modes with $b / a$ were then studied. It is interesting to see that for all existing ring modes, there is a b/a value (around 0.7) leading to the maximum coupling coefficient amplitude. With the solid ring thickness too large or too small, coupling strength is significantly reduced.

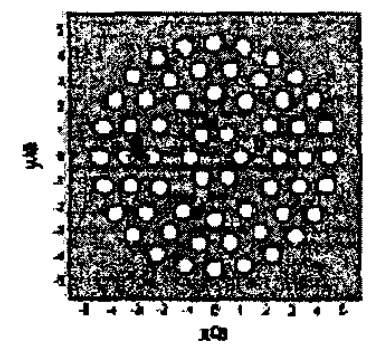

Fig. 1 Structure of the micro-structured fiber.

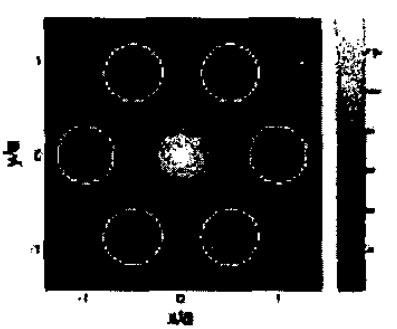

Fig. 2 Intensity distribution of the core mode.

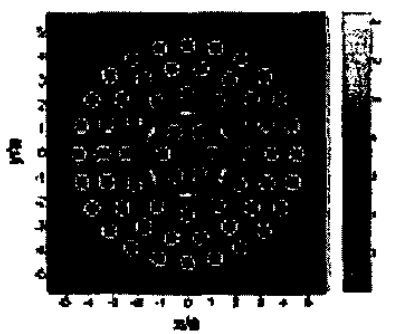

Fig. 3 Intensity distribution of the first ring mode. 